\title{
Comparison of clinical features and prognostic factors in HIV-negative adults with cryptococcal meningitis and tuberculous meningitis: a retrospective study
}

\author{
Junyan Qu, Taoyou Zhou, Cejun Zhong, Rong Deng and Xiaoju Lü*
}

\begin{abstract}
Background: The incidence of cryptococcal meningitis (CM) and tuberculous meningitis (TBM) have gradually increased in recent years. These two types of meningitis are easily misdiagnosed which leads to a poor prognosis. In this study we compared differences of clinical features and prognostic factors in non-HIV adults with CM and TBM.

Methods: We retrospectively reviewed the medical records of CM and TBM patients from January 2008 to December 2015 in our university hospital in China. The data included demographic characteristics, laboratory results, imaging findings, clinical outcomes.

Results: A total of 126 CM and 105 TBM patients were included. CM patients were more likely to present with headache, abnormal vision and hearing, and they might be less prone to fever and cough than TBM patients $(P<0.05)$. Higher percentage of $C M$ patients presented with cerebral ischemia/infarction and demyelination in brain MRI than TBM patients $(P<0.05)$. CM patients had lower counts of WBC in CSF, lower total protein in CSF and serum CD4/CD8 ratio than TBM patients $(P<0.05)$. After three months of treatment, $C M$ group have worse outcome than TBM group $(P<0.05)$. Multivariate analysis showed that age more than 60y $(\mathrm{OR}=4.981,95 \% \mathrm{Cl}: 1$. 955-12.692, $P=0.001)$, altered mentation $(\mathrm{OR}=5.054,95 \% \mathrm{Cl}: 1.592-16.046, P=0.006), \mathrm{CD} 4 / \mathrm{CD} 8$ ratios $<1(\mathrm{OR}=8$. 782, 95\% Cl: 2.436-31.661, $P=0.001)$ and CSF CrAg $\geq 1: 1024$ (OR=4.853, 95\% Cl: 1.377-17.098, $P=0.014)$ were independent risk factors for poor prognosis for CM patients. For TBM patients, hydrocephalus (OR =7.290, 95\% Cl: 1 . $630-32.606, P=0.009)$ and no less than three underlying diseases $(\mathrm{OR}=6.899,95 \% \mathrm{Cl}: 1.766-26.949, P=0.005)$ were independent risk factors, headache was a protective factor of prognosis.
\end{abstract}

Conclusions: Our study provided some helpful clues in the differential diagnosis of non-HIV patients with CM or TBM and identified some risk factors for the poor prognosis of these two meningitis which could help to improve the treatment outcome. Further studies are worth to be done.

Keywords: Cryptococcal meningitis, Tuberculous meningitis, Clinical features, Prognostic factors, HIV negative

* Correspondence: Ivxj33966@126.com

Center of Infectious Disease, West China Hospital, Sichuan University, 37

Guoxue Lane, Chengdu 610041, China International License (http://creativecommons.org/licenses/by/4.0/), which permits unrestricted use, distribution, and reproduction in any medium, provided you give appropriate credit to the original author(s) and the source, provide a link to the Creative Commons license, and indicate if changes were made. The Creative Commons Public Domain Dedication waiver (http://creativecommons.org/publicdomain/zero/1.0/) applies to the data made available in this article, unless otherwise stated. 


\section{Background}

Many organisms such as meningococcus, Mycobacterium tuberculosis and Cryptococcus neoformans can result in meningitis. With increasing use of immune suppressive medication use in rheumatologic conditions, oncology, and organ transplantation, the incidence of Cryptococcal meningitis $(\mathrm{CM})$ and tuberculous meningitis (TBM) are rising $[1,2]$.

$\mathrm{CM}$ and TBM are the two most common types of chronic infectious meningitis, especially in developing countries, and may have similar clinical manifestations and cerebrospinal fluid (CSF) findings [3, 4]. Although mortality has decreased significantly in recent years, with the advent of new antimicrobial drugs, diagnostic techniques and treatment strategies, it remains high $[3,5-7]$. $\mathrm{CM}$ and TBM are easily misdiagnosed due to vague clinical syndromes associated with these conditions $[3,6]$. Delay of diagnosis and treatment are directly related to a poor prognosis $[3,8]$. Early diagnosis CM and TBM, especially TBM, remain challenges for clinicians.

$\mathrm{CM}$ and TBM are common in human immunodeficiency virus (HIV) infected patients, but they are also seen in patients with other forms of immunosuppression and apparently immunocompetent individuals [9]. Some studies have sought to identify clinical features in order to distinguish TBM from CM in human HIV infected patients $[10,11]$. Data on how to identify CM and TBM according to clinical profiles in HIV negative patients are scarce. In this retrospective study, we aimed to compare the demographic features, clinical presentations, laboratory data, radiographic findings, therapeutic outcomes and prognostic factors of HIV negative TBM and $\mathrm{CM}$ patients who were admitted to a university hospital from 2008 to 2015 in China.

\section{Methods}

\section{Study population}

The retrospective study was conducted between January 2008 and December 2015, in West China Hospital, Sichuan University, China (a 4,300-bed academic tertiary care hospital). Patients aged 14 years or older with confirmed diagnosis of $\mathrm{CM}$ or clinical or confirmed diagnosis of TBM were included. Exclusion criteria were age under 14 years, HIV positive with infected, pregnant mothers, CM combined with TBM, and cases lacking follow-up for anti-tuberculosis or anti-fungal treatment.

The diagnosis of CM and TBM were made by clinical and laboratory findings. Symptoms and signs of meningitis including one or more of the following: headache, vomiting, fever, neck stiffness, convulsions, focal neurological deficits, altered mentation. Laboratory assessments for CM included CSF culture, CSF India ink testing, and CSF cryptococcal antigen testing ( $\mathrm{CrAg}$ ). Patients with TBM included definite TBM and clinical diagnosis of TBM. The diagnostic criteria of definite TBM was clinical findings plus one or more of the following: acid-fast bacilli seen in the CSF, M. tuberculosis cultured from the CSF, or a CSF positive commercial nucleic acid amplification test. The clinical diagnosis of TBM based on the clinical algorithm [12], which modified according to Marais' definitions [13]. All enrolled patients were divided into CM and TBM groups.

Altered mentation in CM and TBM refers to general changes in brain function, such as confusion, amnesia, loss of alertness, loss of orientation, defects in judgment and unusual behavior. Which were confirmed by psychiatrists.

\section{Laboratory studies}

Laboratory tests such as complete blood counts, blood biochemistry, interferon gamma release assays (IGRAs), peripheral blood $\mathrm{T}$ lymphocyte subset measurements and HIV test were done at the department of laboratory medicine of our hospital. Lumbar puncture (LP) and tuberculin skin test (TST) of suspected CM or TBM cases were performed before initial treatment. CSF was sent for WBC, glucose, protein, chloride, India ink stain, CSF cryptococcal antigen titers, TB smear, TB PCR, fungal and $M$. tuberculosis cultures. These tests were done at the discretion of the treating physicians. Fluorescence quantitative PCR was used to detect TB DNA in CSF. The rapid liquid culture mediums were used to detect M. Tuberculosis. Fungal culture of CSF was performed on Sabouraud dextrose agar (SDA) and incubated at $30^{\circ} \mathrm{C}$ to obtain cryptococcus isolates. The CSF cryptococcal antigen titers were determined by Latex Cryptococcal Antigen Detection System (Immuno-Mycologics, Inc., Norman, USA). The test utilizes latex particles coated with anti-cryptococcal antibody, which will reacts with the cryptococcal capsular polysaccharide antigen causing a visible agglutination. Brain magnetic resonance imaging (MRI) or computed tomography (CT) examinations and chest CT were performed at the discretion of the physicians.

\section{Treatment strategies}

All the patients received treatments after they got the diagnoses. Time of diagnosis was the time from onset of symptoms to treatment. HIV negative CM patients were treated with amphotericin B (0.7-1 $\mathrm{mg} / \mathrm{kg} / \mathrm{d})$, 5flucytosine $(100 \mathrm{mg} / \mathrm{kg} / \mathrm{d})$ with or without fluconazole $(800 \mathrm{mg} /$ day)/voriconazole (loading dose, $6 \mathrm{mg} / \mathrm{kg} / 12 \mathrm{~h}$, maintenance dose, $4 \mathrm{mg} / \mathrm{kg} / 12 \mathrm{~h}$ ) for $6-12$ weeks of induction therapy, and then fluconazole ( $400 \mathrm{mg} /$ day) for 8 weeks of consolidation therapy, fluconazole $(200 \mathrm{mg} /$ day) for more than 6 months of maintenance therapy according to the guidelines for the management of cryptococcal disease and our experiences [5, 14]. Only three patients with $\mathrm{CM}$ received voriconazole. Because 
amphotericin B was contraindicated in some patients with kidney disease or renal inadequacy emerged after use of amphotericin B in another patient. Continuous lumbar drainage subarachnoid were applied to the CM patients with persistent high intracranial pressure. The treatment regimen for TBM patients who had not been treated with chemotherapy consisted of three months of isoniazide (INH), rifampin (RIF), pyrazinamide (PZA), and streptomycin $(\mathrm{SM}) /$ ethambutol (EMB), followed by INH and RIF $[15,16]$. The total treatment course was at least 12 month. Second-line treatment regimens were given after failure to first-line TB chemotherapy according to the guideline and patients' situation $[15,16]$.

\section{Data collection}

We retrospectively reviewed the medical records of patients involved in this study. We obtained demographic characteristics, underlying diseases, use of steroids, symptoms and signs on admission, duration of initial symptoms to diagnosis or treatment, laboratory data, brain MRI /CT findings and clinical outcomes. The clinical outcomes were evaluated after three-month of treatment. Risk factors for the prediction of poor of HIV negative CM and TBM patients were also evaluated. All data were verified by two authors independently.

\section{Ethical considerations}

The study was approved by the Ethics Committee of West China Hospital, Sichuan University, which waived the need for informed consent because all the data used in this study were routinely obtained and no additional procedures were carried out.

\section{Statistical analysis}

Statistical analyses were performed using SPSS version 17.0 software package (SPSS Inc., Chicago, USA). The Shapiro-Wilk normality test was used for testing normality for all quantitative variables. Continuous variables were presented as mean \pm standard deviation or median with interquartile ranges (IQR). Signifcance testing was carried out using Student's $t$ test for normally distributed data and Mann-Whitney $U$ test for non-normal data. Chi-square test or Fisher exact test was used for categorical variables. Risk factors for three-month treatment outcomes of CM or TBM were identified using a logistic regression model. All variables were initially tested by univariate analysis and those with a $P<0.05$ were entered stepwise into multivariate analysis by using the forward conditional method. Then the regression equations for predicting the probability of poor prognosis of $\mathrm{CM}$ or TBM were established. Receiver operating characteristic (ROC) curves were plotted for these two predicted models with the Medcalc software version 11.5.1.0 (Medcalc software bvba, Belgium). Predictive accuracy was assessed by calculating the areas under the ROC curves (AUC). An AUC of $>0.8$ was considered very good. A two-tailed $P<0.05$ was considered statistically significant.

\section{Results}

\section{Patient characteristics}

From January 2008 to December 2015, a total of 126 $\mathrm{CM}$ patients and 105 TBM patients without HIV infection were included in this retrospective study. The clinical characteristics of these patients were shown in Table 1. There was no significant difference between $\mathrm{CM}$ and TBM patients in gender.

Most patients with CM were from 30 to 60 years (65/ $126,51.6 \%)$, but most of TBM patients were aged under 30 years $(54 / 105,51.4 \%)$. Among CM patients, the most common initial symptoms were headache (118/126, 96.7\%), followed by fever (71/126, 56.3\%), vomiting (71/126, 56.3\%) and altered mentation (51/126, 40.5\%). The most common presenting symptoms of TBM patients were also headache (87/105, 82.9\%), fever (72/105, 68.6\%) and vomiting (47/ $105,44.8 \%)$. CM patients were more likely to present with headache, abnormal vision and hearing, and less likely to present with fever and cough than TBM patients $(P<0.05)$.

The most frequently underlying diseases, factors or complications of CM patients were corticosteroid use (22/ $126,17.5 \%)$, pulmonary infection $(22 / 126,17.5 \%)$, hepatobiliary diseases $(15 / 126,11.9 \%)$ and diabetes mellitus (13/ 126, 10.3\%). However, tuberculosis outside CNS was the most common in TBM patients, especially disseminated tuberculosis $(27 / 62,43.5 \%$, data not shown). Twenty percent of patients had no underlying condition.

\section{Laboratory data and radiological findings}

Laboratory data and radiographic findings are prior to shown in Table 2. The CSF WBC and total protein in CM patients were lower than those of TBM group $(P<0.05)$. There was no statistical difference in CSF glucose, chloride level and opening pressure in LP between the two groups. In HIV negative CM group, CD4/CD8 ratio in serum was significantly decreased compared to the TBM group.

Brain MRI was performed in 84.9\% (107/126) patients with CM and 76.2\% (80/105) patients with TBM. Other patients were examined by head CT. The most common manifestations were cerebral ischemia, demyelination, meningeal enhancement and encephalitis in these two groups. A total of $15.9 \%(20 / 126)$ patients in CM group and $11.4 \%(12 / 105)$ in TBM group had normal image findings. A higher percentage of $\mathrm{CM}$ patients presented with cerebral ischemia/infarction and demyelination than TBM patients $(P<0.05)$, but encephalitis and cerebral edema were more common in TBM patients $(P<0.05)$. 
Table 1 Demographic and clinical features in HIV negative patients with cryptococcal meningitis (CM) and tuberculous meningitis (TBM)

\begin{tabular}{|c|c|c|c|}
\hline Variables & $C M(N=126)$ & TBM $(N=105)$ & $P$-value \\
\hline \multicolumn{4}{|l|}{ Sex } \\
\hline Male & $79(62.7 \%)$ & $57(54.3 \%)$ & \multirow[t]{2}{*}{0.227} \\
\hline Female & $47(37.3 \%)$ & $48(45.7 \%)$ & \\
\hline \multicolumn{4}{|l|}{ Age (years) } \\
\hline$\leq 30$ & $29(23.0 \%)$ & $54(51.4 \%)$ & \multirow[t]{3}{*}{$<0.001$} \\
\hline $31-60$ & $65(51.6 \%)$ & 35(33.3\%) & \\
\hline$>60$ & $32(25.4 \%)$ & $16(15.2 \%)$ & \\
\hline \multicolumn{4}{|l|}{ Presenting symptoms and signs } \\
\hline Headache & $118(96.7 \%)$ & 87(82.9\%) & 0.012 \\
\hline Fever & $71(56.3 \%)$ & $72(68.6 \%)$ & 0.038 \\
\hline Vomiting & $71(56.3 \%)$ & $47(44.8 \%)$ & 0.263 \\
\hline Altered mentation & $51(40.5 \%)$ & $43(41.0 \%)$ & 0.524 \\
\hline Abnormal vision & $35(27.8 \%)$ & $12(11.4 \%)$ & 0.002 \\
\hline Cough & $7(5.6 \%)$ & $24(22.9 \%)$ & $<0.001$ \\
\hline Seizure & 14(11.1\%) & $6(5.7 \%)$ & 0.111 \\
\hline Abnormal hearing & 14(11.1\%) & $3(2.9 \%)$ & 0.014 \\
\hline Apopsychia & $6(4.8 \%)$ & $2(1.9 \%)$ & 0.208 \\
\hline Dyspnea & $5(4.0 \%)$ & $1(1.0 \%)$ & 0.155 \\
\hline Meningeal signs & $89(70.6 \%)$ & $68(64.8 \%)$ & 0.209 \\
\hline Pathological signs & $22(17.5 \%)$ & $23(21.9 \%)$ & 0.247 \\
\hline \multicolumn{4}{|c|}{ Underlying diseases, factors or complications } \\
\hline Corticosteroid medication & $22(17.5 \%)$ & $6(5.7 \%)$ & 0.005 \\
\hline Pnumonary infection & $22(17.5 \%)$ & $13(12.4 \%)$ & 0.188 \\
\hline Hepatobiliary diseases & $15(11.9 \%)$ & $12(11.4 \%)$ & 0.539 \\
\hline Tuberculosis outside CNS & $12(9.5 \%)$ & $62(59.0 \%)$ & $<0.001$ \\
\hline Immune system disease & $11(8.7 \%)$ & $6(5.7 \%)$ & 0.269 \\
\hline Hypertension & $11(8.7 \%)$ & $3(2.9 \%)$ & 0.054 \\
\hline Diabetes mellitus & 13(10.3\%) & $10(9.5 \%)$ & 0.510 \\
\hline Chronic lung disease & $6(4.8 \%)$ & $3(2.9 \%)$ & 0.348 \\
\hline $\begin{array}{l}\text { Multiple organ dysfunction } \\
\text { syndrome }\end{array}$ & $6(4.8 \%)$ & $1(1.0 \%)$ & 0.095 \\
\hline Renal transplantation & $4(3.2 \%)$ & $2(1.9 \%)$ & 0.431 \\
\hline Hematology disease & $3(2.4 \%)$ & $1(1.0 \%)$ & 0.382 \\
\hline Others & 13(10.2\%) & $12(11.4 \%)$ & 0.571 \\
\hline None & $29(23.0 \%)$ & $20(19.0 \%)$ & 0.284 \\
\hline \multicolumn{4}{|c|}{ Time from onset of symptoms to treatment (d) } \\
\hline $0-30$ & 48(38.1\%) & $56(53.3 \%)$ & \multirow[t]{4}{*}{$<0.001$} \\
\hline $31-60$ & $47(37.3 \%)$ & $33(31.4 \%)$ & \\
\hline $61-90$ & 19(15.1\%) & $13(12.4 \%)$ & \\
\hline$>90$ & $12(9.5 \%)$ & $3(2.9 \%)$ & \\
\hline Outcome Improved & $80(63.5 \%)$ & $81(77.1 \%)$ & \multirow[t]{2}{*}{0.031} \\
\hline Worse/Died & $46(36.5 \%)$ & $24(22.9 \%)$ & \\
\hline
\end{tabular}

CM and TBM specific test results are shown in Table 3. For CM patients, latex agglutination $\mathrm{CrAg}$ titer were positive in $99.2 \%(125 / 126)$ of them. The positive rates of CSF India ink stain and CSF culture for C. neoformans in CM patients were $77.8 \%(98 / 126)$ and $79.3 \%$ (100/126), respectively. IGRA had higher positive rate than other detection methods in TBM patients $(P<0.05)$.

\section{Treatment outcome}

A total of 38.1 and $53.3 \%$ of patients with CM and TBM received treatment within 30 days after the onset of clinical symptoms, respectively. And the proportion of patients with a duration of more than 2 month was higher in the CM group than TBM group (26.6\% vs. $15.2 \%$, $P=0.100)$, but there was no significant difference. After three months of treatment, $63.5 \%$ (80/126) of CM patients and $77.1 \%(81 / 105)$ of TBM patients had improved $(P=0.031)$.

\section{Risk factors for poor treatment outcome of HIV negative CM and TBM patients}

Univariate analysis revealed that the factors impacting the prognosis of $\mathrm{CM}$ patients were age, altered mentation, demyelination, CD4/CD8 ratios $<1$ and CSF $\mathrm{CrAg} \geq 1: 1024$. Multivariate analysis showed that age, altered mentation, CD4/CD8 ratios $<1$ and CSF CrAg $\geq$ 1:1024 were independent risk factors for poor prognosis. For TBM patiens, multiple regression analysis showed that hydrocephalus $(\mathrm{OR}=7.290,95 \% \mathrm{CI}: 1.630-32.606$, $P=0.009)$ and no less than three underlying diseases (OR $=6.899,95 \% \mathrm{CI}: 1.766-26.949, P=0.005)$ were the risk factors, and headache was a protective factor of prognosis, as shown in Table 4.

\section{ROC curve for the prognostic index derived from the logistic regression models}

Prediction models for the probability of poor prognosis in patients with CM and TBM are shown in Appendix 1. The ROC curves of the models of CM and TBM are shown in Additional file 1: Figure S1.

\section{Discussion}

Many patients with CM and TBM were misdiagnosed or had a delay in diagnosis. Fever, headache, vomiting, altered mentation and meningeal irritation were the most common initial symptoms of both CM and TBM patients. However, CM patients were more likely to suffer headache, vision and hearing damage, TBM patients were more prone to have fever and cough. These results were fairly consistent with previous reports $[9,17,18]$. One review showed headache (87\%), fever (74\%), meningeal irritation (67\%), vomiting (61\%) and altered mentation (26\%) as the most common clinical manifestations of CM [9]. One study showed that among 
Table 2 Laboratory data and radiographic findings in HIV negative patients with cryptococcal meningitis (CM) and tuberculous meningitis (TBM)

\begin{tabular}{|c|c|c|c|}
\hline Variables & $C M(N=126)$ & $\operatorname{TBM}(N=105)$ & $P$-value \\
\hline \multicolumn{4}{|l|}{ CSF } \\
\hline WBC (cells/mm³), Median (IQR) & $80.00(20.00-200.00)$ & $120.00(59.00-295.00)$ & 0.005 \\
\hline Total protein (mg/dL), Median (IQR) & $0.83(0.58-1.32)$ & $1.53(0.99-2.66)$ & $<0.001$ \\
\hline Glucose (mmol/L), Median (IQR) & $1.57(0.80-2.56)$ & $1.78(1.10-2.35)$ & 0.694 \\
\hline Chloride (mmol/L), (mean \pm SD) & $118.11 \pm 6.67$ & $111.40 \pm 7.59$ & 0.169 \\
\hline Open pressure $>220 \mathrm{~mm} \mathrm{H}_{2} \mathrm{O}$ & $98(77.78 \%)$ & $75(71.4 \%)$ & 0.289 \\
\hline \multicolumn{4}{|l|}{ Serum } \\
\hline Albumin(g/L), (mean $\pm S D)$ & $37.77 \pm 5.29$ & $36.61 \pm 5.20$ & 0.688 \\
\hline WBC $\left(\times 10^{9} / \mathrm{L}\right),($ mean $\pm \mathrm{SD})$ & $8.89 \pm 3.51$ & $7.46 \pm 3.45$ & 0.701 \\
\hline Neutrophile (\%) & $78.44 \pm 10.85$ & $75.92 \pm 11.33$ & 0.690 \\
\hline Hemoglobin (g/L), (mean $\pm S D)$ & $122.83 \pm 23.05$ & $118.50 \pm 22.71$ & 0.548 \\
\hline $\mathrm{CD}^{+}$T lymphocyte (\%) & $65.11 \pm 14.25$ & $70.96 \pm 13.20$ & 0.533 \\
\hline CD4+ T lymphocyte (\%) & $28.71 \pm 10.46$ & $34.77 \pm 11.27$ & 0.542 \\
\hline CD8 ${ }^{+}$T lymphocyte (\%) & $25.40 \pm 8.57$ & $25.19 \pm 10.26$ & 0.139 \\
\hline CD4/CD8 ratio & $1.22 \pm 0.59$ & $1.71 \pm 1.12$ & 0.003 \\
\hline \multicolumn{4}{|l|}{ Brain images (CT/MRI) } \\
\hline Cerebral ischemia/infarction & $55(43.7 \%)$ & $30(28.6 \%)$ & 0.028 \\
\hline Demyelination & $30(23.8 \%)$ & $14(9.5 \%)$ & 0.046 \\
\hline Meningeal enhancement & $23(18.3 \%)$ & $20(16.0 \%)$ & 0.877 \\
\hline Hydrocephalus & $16(12.7 \%)$ & $17(16.2 \%)$ & 0.709 \\
\hline Encephalitis & $14(11.1 \%)$ & $40(38.1 \%)$ & $<0.001$ \\
\hline Cerebral edema & $4(3.17 \%)$ & $13(12.4 \%)$ & 0.010 \\
\hline Others & $4(3.17 \%)$ & $8(7.6 \%)$ & 0.147 \\
\hline Normal & 20 (15.9\%) & $12(11.4 \%)$ & 0.347 \\
\hline
\end{tabular}

CSF cerebrospinal fluid, $W B C$ white blood count, $M R I$ magnetic resonance imaging

patients without HIV, those with CM were more likely to present headache, altered mentation, vision and hearing damage as compared to those with TBM [18].

Although CM and TBM are often considered as an opportunistic infection in patients with HIV, they are also seen in individuals with apparently normal immune function. In this study, corticosteroid medication, hepatobiliary diseases (abnormal liver function for various reasons, such as chronic hepatitis, cirrhosis) and diabetes mellitus occupied the top three underlying diseases and factors for CM patients. A study reviewed 3698 cryptococcosis cases that found the most common underlying diseases were HIV infection, tuberculosis, liver disease, systemic lupus erythematosus and diabetes mellitus, while $17 \%$ had no underlying diseases [9]. Another study showed the commom underlying disease of HIV negative CM patients were hepatobiliary diseases, hypertension, cancer and diabetes mellitus [7]. Sixty-two TBM patients, 27 of them were disseminated tuberculosis, in this study were known to have pulmonary

Table 3 The positive rate of pathogens and other serological tests for diagnosis of cryptococcal meningitis (CM) and tuberculous meningitis (TBM) in HIV negative patients

\begin{tabular}{llll}
\hline Variables & CM (N=126) & Variables & TBM (N=105) \\
\hline India ink stain & $98(77.8 \%)$ & TB smearing & $5(4.8 \%)$ \\
CSF fungal culture & $100(79.3 \%)$ & TB DNA & $59(56.2 \%)$ \\
India ink stain \& fungal culture & $72(57.1 \%)$ & Mycobacterium tuberculosis culture & $9(8.6 \%)$ \\
CrAg & $125(99.2 \%)$ & IGRA & $94(89.5 \%)$ \\
CrAg titer $\geq 1: 1024$ & $73(57.9 \%)$ & TST & $62(60.0 \%)$ \\
\hline
\end{tabular}

CSF cerebrospinal fluid, CrAg cryptococcal antigen, TST tuberculin skin test, IGRA interferon gamma release assay 
Table 4 Risk factors associated with poor prognosis of HIV negative cryptococcal meningitis (CM) and tuberculous meningitis (TBM) patients

\begin{tabular}{|c|c|c|c|c|c|c|}
\hline \multicolumn{7}{|l|}{ Cryptococcal meningitis (CM) } \\
\hline \multirow[t]{2}{*}{ Variables } & \multicolumn{3}{|c|}{ Univariate analysis } & \multicolumn{3}{|c|}{ Multivariate analysis } \\
\hline & Odds ratio & $95 \% \mathrm{Cl}$ & $P$-value & Odds ratio & $95 \% \mathrm{Cl}$ & $P$-value \\
\hline Ages $>60 y$ & 5.946 & $2.493-14.183$ & $<0.001$ & 4.981 & $1.955-12.692$ & 0.001 \\
\hline Altered mentation & 4.533 & $1.763-11.656$ & 0.002 & 5.054 & $1.592-16.046$ & 0.006 \\
\hline Demyelination & 7.270 & $2.560-20.646$ & $<0.001$ & & & \\
\hline CD4/CD8 ratio $<1$ & 8.475 & $2.831-25.378$ & $<0.001$ & 8.782 & $2.436-31.661$ & 0.001 \\
\hline CrAg titer $\geq 1: 1024$ & 5.338 & $1.769-16.103$ & 0.003 & 4.853 & $1.377-17.098$ & 0.014 \\
\hline \multicolumn{7}{|l|}{ Tuberculous meningitis (TBM) } \\
\hline Fever & 9.659 & $1.208-77.252$ & 0.033 & & & \\
\hline Headache & 0.139 & $0.042-0.459$ & 0.001 & 0.125 & $0.032-0.497$ & 0.003 \\
\hline Hydrocephalus & 3.692 & $1.062-12.839$ & 0.040 & 7.290 & $1.630-32.606$ & 0.009 \\
\hline Underlying diseases or factors $\geq 3$ & 6.817 & $1.927-19.869$ & 0.002 & 6.899 & $1.766-26.949$ & 0.005 \\
\hline
\end{tabular}

HIV human immunodeficiency virus, $\mathrm{CrAg}$ cryptococcal antigen, $\mathrm{Cl}$ confidential intervals

tuberculosis. Gu et al. also found 30.1\% of TBM patients derived their TBM from disseminated tuberculosis [17]. TBM is the most severe form of extrapulmonary tuberculosis, it can occur in isolation or along with a pulmonary focus. TBM and disseminated tuberculosis are usually due to hematogenous dissemination of the tubercle bacillus [19].

In CM and TBM patients, the most common manifestations on brain imagines were cerebral ischemia/infarction, meningeal enhancement, hydrocephalus, which consistent with previous reports $[3,5,20]$. Demyelination were seen on some patients' head MRI or CT. Of course, MR imaging is more sensitive in depicting demyelination than head CT. In this study, demyelination was more commom for $\mathrm{CM}$ patients than in prior reports $[5,20]$. Another study showed that gadoliniumenhancing white matter lesions found on brain MRI of CM patients. Biopsy revealed the lesions of resembled demyelinating were cryptococci, non-specific inflammatory changes or small perivascular lymphocyte collections. Which possibly be leukoencephalopathy or immune response to the pathogen [21]. In addition, our patients showed evidence of cerebral ischemia or encephalitis more often than previous studies $[9,18]$, this was likely related to the increased sensitivity of MRI with diffusion-weighted imaging.

Patients with TBM presented with higher CSF WBC and total protein than $\mathrm{CM}$ patients, similar to prior studies [18]. A low ratio of CD4/CD8 was also found in the apparently healthy $\mathrm{CM}$ patients, it's possible those patients may have some undetectable underlying disease.

In our study, multivariate analysis showed that age $>60$ years, altered mentation, CD4/CD8 ratios $<1$ and CSF $\mathrm{CrAg} \geq 1: 1024$ were closely correlated with poor outcome of CM patients. Previous studies showed there were many poor prognostic factors for $\mathrm{CM}$, including Cryptococcus growth from sites other than CSF, altered mentation, intracranial pressure (ICP) $>400 \mathrm{mmH}_{2} \mathrm{O}$, CSF glucose $<40$ $\mathrm{mg} / \mathrm{dl}$, high CSF CrAg titer, decreased CSF/blood glucose ratio, papilledema, chronic use of corticosteroids or other immunosuppressants, absence of headache and low GCS score [7, 20, 22-24]. Our study showed that CrAg test was highly specific to the diagnosis of cryptococcal meningitis, and it also had prognostic value, similar to previous studies [25]. Though middle-aged people made up a greater percentage of our CM patients, mortality was increased with patients aged greater than than 60 years, possibly related to a decreased immune response to pathogens declines with age [26].

For TBM patients, poor outcome was related to hydrocephalus and more than three underlying diseases in the present study. Hydrocephalus, optochiasmatic arachnoiditis, microbiologically confirmed TBM have been associated with poor outcome of TBM in previous studies $[27,28]$. Early diagnosis and treatment improve prognosis. We found no literature showing that more than three underlying diseases was correlated with poor outcome of TBM. It might be because these studies did not include this factor. On admission, there were $17.1 \%$ TBM patients had no headache. Headache was a protective factor for TBM in this study. The inflammation that occurs in the subarachnoid space during meningitis can largely be attributed to the response of the immune system to bacteria, which leads to headache [29]. Patients without headache probably means they have weak immune response. So they had poor outcome.

There were some limitations concerning this study. Because our study was a retrospective research, the medical records about epidemiological contact history were incomplete, so we did not analyse relevant 
contents. In addition, there were three patients with $\mathrm{CM}$ using voriconazole because they could not tolerate the adverse effect of amphotericin B or they complicated renal disease. But just three patients used non standard treatments, which might not affect the statistic results.

\section{Conclusions}

We found that compared to patients with TBM, CM patients were more likely to present with headache, vision and hearing damage, cerebral ischemia and demyelination. TBM patients were more likely to have fever, cough, encephalitis and cerebral edema. CM patients had lower CSF WBC, total protein, and CD4/ CD8 ratio than those of TBM patients. Prognosis was poorer for $\mathrm{CM}$ patients than TBM patients. Age, altered mentation, CD4/CD8 ratios $<1$ and CSF $\mathrm{CrAg} \geq$ 1:1024 were independent risk factors for poor prognosis of HIV negative CM patients. Hydrocephalus and no less than three underlying diseases were the risk factors, and headache was a protective factor for HIV negative patients with TBM. Our prediction models may be helpful for making treatment plan and prognosis assessment. Of course, specific identification of the offending microorganism is considered to be the gold standard in diagnosing infectious diseases. More work needs to be done to develop more rapid, sensitive and specific diagnosis methods and more effective treatments for patients CM and TBM, and the prognosis can be improved accordingly.

\section{Appendix 1}

Prediction models for the probability of poor prognosis in patients with cryptococcal meningitis (CM) and tuberculous meningitis (TBM)

Prediction model for the probability of poor prognosis in CM: $P_{C M}=\mathrm{e}^{\mathrm{x}} /\left(1+\mathrm{e}^{\mathrm{x}}\right), \mathrm{x}=-6.360+(1.606 \times$ age $)+$ $(1.620 \times$ altered mentation $)+(2.173 \times \mathrm{CD} 4 / \mathrm{CD} 8$ ratios $)$ $+(1.579 \times$ CSF CrAg titer $)$. Prediction model for the probability of poor prognosis in TBM: $P_{T B M}=\mathrm{e}^{\mathrm{x}} /\left(1+\mathrm{e}^{\mathrm{x}}\right)$, $\mathrm{x}=-0.658+(1.931 \times$ underlying diseases $)+(1.987 \times$ hydrocephalus $)+(-2.079 \times$ headache $)$. Where the following are used: $e$ is the natural logarithm; age $>60 \mathrm{y}$, altered mentation, CD4/CD8 ratios $<1$, CSF $\mathrm{CrAg}$ titer $\geq 1: 1024$, underlying diseases $\geq 3$ and headache are scored as 1 or otherwise as 0 ; hydrocephalus was derived from the imaging report (1: yes, 0 : no). The ROC curves of the risk index models for predicting poor prognosis of CM or TBM are shown in Additional file 1: Figure S1. The AUC of CM was 0.872 (95\% CI: 0.786-0.932). The appropriate cut off point was selected at $\mathrm{P}_{\mathrm{CM}}=0.5127$, and the model achieved a sensitivity of $69.44 \%$, a specificity of $91.23 \%$. The AUC of TBM was 0.802 (95\% CI: $0.700-0.882)$, the appropriate cut off point was 0.3209 for $\mathrm{P}_{\mathrm{TBM}}$ with a sensitivity of $57.89 \%$ and a specificity of $88.89 \%$.

\section{Additional file}

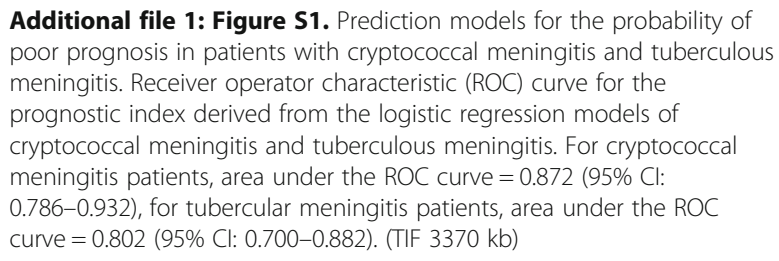

Additional file 1: Figure S1. Prediction models for the probability of poor prognosis in patients with cryptococcal meningitis and tuberculous meningitis. Receiver operator characteristic (ROC) curve for the prognostic index derived from the logistic regression models of cryptococcal meningitis and tuberculous meningitis. For cryptococcal meningitis patients, area under the ROC curve $=0.872(95 \% \mathrm{Cl}$ : 0.786-0.932), for tubercular meningitis patients, area under the ROC curve $=0.802(95 \% \mathrm{Cl}: 0.700-0.882)$. (TIF $3370 \mathrm{~kb})$

\section{Abbreviations}

5-FC: 5-flucytosine; AIDS: Acquired immune deficiency syndrome; AMB: Amphotericin B; CM: Cryptococcal meningitis; CNS: Central nervous system; CrAg: Cryptococcal antigen; CSF: Cerebrospinal fluid; CT: Computed tomography; EMB: Ethambutol; HIV: Human immunodeficiency virus; IGRA: Interferon gamma release assay; INH: Isoniazide; LP: Lumbar puncture; MRI: Magnetic resonance imaging; PCR: Polymerase chain reaction; PZA: Pyrazinamide; RIF: Rifampin; TBM: Tuberculous meningitis;

TST: Tuberculin skin test; WBC: White blood cell count

\section{Acknowledgments}

The authors thank the staff in Information Center of West China Hospital, Sichuan University for data retrieval. The authors would like to thank Dr Hui Ye for helpful in study design and discussion.

\section{Funding}

This research received no specific grant from any funding agency in the public, commercial, or not-for-profit sectors.

\section{Availability of data and materials}

Aggregated data is entirely presented in this publication. Individual data will not be shared following the ethical principle of confidentiality in the use of patient data.

\section{Authors' contributions}

XJL had full access to all the data in the study and takes responsibility for the integrity of the data and the accuracy of the analysis and will act as the guarantor. All authors participated in study conception, design and interpretation. JYQ wrote the initial draft. TYZ revised the manuscript critically for important intellectual content. CJZ and RD participated in acquisition and analysis of data, and critical review. All authors have seen and approved the final version

\section{Competing interests}

The authors declare that they have no competing interests.

\section{Consent for publication}

Not applicable.

\section{Ethics approval and consent to participate}

The study was approved by the Ethics Committee of West China Hospital, Sichuan University, which waived the need for informed consent because all the data used in this study were routinely obtained and no additional procedures were carried out

Received: 9 August 2016 Accepted: 14 December 2016

Published online: 10 January 2017

\section{References}

1. Christensen AS, Andersen AB, Thomsen VO, Andersen PH, Johansen IS. Tuberculous meningitis in Denmark: a review of 50 cases. BMC Infect Dis. 2011;11:47.

2. Pyrgos V, Seitz AE, Steiner CA, Prevots DR, Williamson PR. Epidemiology of cryptococcal meningitis in the US: 1997-2009. Plos One. 2013;8(2):390-6. 
3. Thwaites GE, van Toorn R, Schoeman J. Tuberculous meningitis: more questions, still too few answers. Lancet Neurol. 2013;12(10):999-1010.

4. Lee HG, William T, Menon J, Ralph AP, Ooi EE, Hou Y, et al. Tuberculous meningitis is a major cause of mortality and morbidity in adults with central nervous system infections in Kota Kinabalu, Sabah, Malaysia: an observational study. BMC Infect Dis. 2016;16:296.

5. Perfect JR, Dismukes WE, Dromer F, Goldman DL, Graybill JR, Hamill RJ, et al. Clinical practice guidelines for the management of cryptococcal disease: 2010 update by the infectious diseases society of America. Clin Infect Dis. 2010;50(3):291-322.

6. Yao Y, Zhang JT, Yan B, Gao T, Xing XW, Tian CL, et al. Voriconazole: a novel treatment option for cryptococcal meningitis. Infect Dis (Lond). 2015:47(10):694-700.

7. Lee YC, Wang JT, Sun HY, Chen YC. Comparisons of clinical features and mortality of cryptococcal meningitis between patients with and without human immunodeficiency virus infection. J Microbiol Immunol Infect. 2011:44(5):338-45.

8. Guo J, Zhou J, Zhang S, Zhang X, Li J, Sun Y, et al. A case-control study of risk factors for HIV-negative children with cryptococcal meningitis in Shi Jiazhuang. China BMC Infect Dis. 2012;12:376.

9. Yuchong C, Fubin C, Jianghan C, Fenglian W, Nan X, Minghui Y, et al. Cryptococcosis in China (1985-2010): review of cases from Chinese database. Mycopathologia. 2012;173(5-6):329-35.

10. Cohen DB, Zijlstra EE, Mukaka M, Reiss M, Kamphambale S, Scholing M, et al. Diagnosis of cryptococcal and tuberculous meningitis in a resource-limited African setting. Trop Med Int Health. 2010;15(8):910-7.

11. Patel VB, Singh R, Connolly C, Kasprowicz V, Zumla A, Ndungu T, et al. Comparison of a clinical prediction rule and a LAM antigen-detection assay for the rapid diagnosis of TBM in a high HIV prevalence setting. PLoS One. 2010;5:e15664.

12. Nhu NT, Heemskerk D, DA T d, Chau TT, Mai NT, Nghia HD. Evaluation of GeneXpert MTB/RIF for diagnosis of tuberculous meningitis. J Clin Microbiol. 2014;52(1):226-33.

13. MarMarais S, Pepper DJ, Marais BJ, Török ME. HIV-associated tuberculous meningitis-diagnostic and therapeutic challenges. Tuberculosis (Edinb). 2010;90(6):367-74.

14. Saag MS, Graybill RJ, Larsen RA, Pappas PG, Perfect JR, Powderly WG, et al. Practice guidelines for the management of cryptococcal disease. Infectious Diseases Society of America. Clin Infect Dis. 2000;30(4):710-8.

15. American Thoracic Society. Treatment of tuberculosis. Morb Mortal Wkly Rep. 2003;52(RR-11):1-77.

16. World Health Organization. Treatment of tuberculosis: guidelines. 4th ed. 2010.

17. Gu J, Xiao H, Wu F, Ge Y, Ma J, Sun W. Prognostic factors of tuberculous meningitis: a single-center study. Int J Clin Exp Med. 2015;8(3):4487-93.

18. Zhang B, Lv K, Bao J, Lu C, Lu Z. Clinical and laboratory factors in the differential diagnosis of tuberculous and cryptococcal meningitis in adult HIV-negative patients. Intern Med. 2013;52(14):1573-8.

19. Galimi R. Extrapulmonary tuberculosis: tuberculous meningitis new developments. Eur Rev Med Pharmacol Sci. 2011;15(4):365-86.

20. Xu XG, Pan WH, Bi XL, Fang W, Chen M, Zhu Y, et al. Comparison of clinical features in patients with persistent and nonpersistent cryptococcal meningitis: twelve years of clinical experience in four centers in China. CNS Neurosci Ther. 2013;19(8):625-31.

21. Wilcox RA, Thyagarajan D, Kempster P. Two cases of Cryptococcus meningitis presenting as leukoencephalopathy prior to amphotericin therapy. Eur J Neurol. 2007;14(3):350-2.

22. Zheng $\mathrm{H}$, Li M, Luo Y, Wang D, Yang J, Chen Q, et al. A retrospective study of contributing factors for prognosis and survival length of cryptococcal meningoencephalitis in Southern part of China (1998-2013). BMC Infect Dis. 2015;15:77.

23. Lessells RJ, Mutevedzi PC, Heller T, Newell ML. Poor long-term outcomes for cryptococcal meningitis in rural South Africa. S Afr Med J. 2011;101(4):251-2.

24. Majumder S, Mandal SK, Bandyopadhyay D. Prognostic markers in AIDSrelated cryptococcal meningitis. J Assoc Physicians India. 2011;59:152-4.

25. Lin TY, Yeh KM, Lin JC, Wang NC, Peng MY, Chang FY. Cryptococcal disease in patients with or without human immunodeficiency virus: clinical presentation and monitoring of serum cryptococcal antigen titers. J Microbiol Immunol Infect. 2009:42(3):220-6.

26. Pourgheysari B, Khan N, Best D, Bruton R, Nayak L, Moss PA. The cytomegalovirus-specific CD4+ T-cell response expands with age and markedly alters the CD4+ T-cellrepertoire. J Virol. 2007;81(14):7759-65.
27. Sharma P, Garg RK, Verma R, Singh MK, Shukla R. Incidence, predictors and prognostic value of cranial nerve involvement in patients with tuberculous meningitis: a retrospective evaluation. Eur J Intern Med. 2011;22(3):289-95.

28. Jha SK, Garg RK, Jain A, Malhotra HS, Verma R, Sharma PK. Definite (microbiologically confirmed) tuberculous meningitis: predictors and prognostic impact. Infection. 2015:43(6):639-45.

29. Sáez-Llorens X, McCracken Jr GH. Bacterial meningitis in children. Lancet. 2003;361(9375):2139-48

\section{Submit your next manuscript to BioMed Central and we will help you at every step:}

- We accept pre-submission inquiries

- Our selector tool helps you to find the most relevant journal

- We provide round the clock customer support

- Convenient online submission

- Thorough peer review

- Inclusion in PubMed and all major indexing services

- Maximum visibility for your research

Submit your manuscript at www.biomedcentral.com/submit
) Biomed Central 